\title{
Age differences in efficiency of locomotion and maximal power output in well-trained triathletes
}

\author{
Damian Coleman · James G. Hopker • Louis Passfield
}

Received: 29 September 2014 / Accepted: 8 October 2014 / Published online: 12 November 2014

(C) Springer-Verlag Berlin Heidelberg 2014

To the editor,

Brisswalter et al. (2014) identified in their recent paper that there is limited data available on the efficiency of locomotion in the ageing athletic population. We have also published work in this field (Hopker et al. 2013), and in that process we encountered some fundamental issues of analysis that have not been addressed by this latest publication. Indeed, our paper prompted a 'letter to the editor' by Boning and Pries (2013) where they clearly articulated the pitfalls associated with comparing gross efficiency values that were calculated at different absolute exercise intensities. Brisswalter et al. (2014) utilised $65 \%$ of maximal aerobic power (MAP) to establish the workload to measure gross efficiency in each individual, however, because MAP was different between age groups, this led to differences in the absolute workload at which this measure was taken (the between group range of approximately 159-260 watts). We have also utilised a relative intensity $(60 \%$ MAP) to assess gross efficiency (Hopker et al. 2013), however, to overcome the issues indicated above; we employed a more robust statistical method to compare the gross efficiency ratio between different age groups. Fundamentally when dealing with ratios such as gross efficiency or indeed basic 'change scores' or 'change scores of ratios' as Brisswalter et al. (2014) have presented, there are assumptions that must be met. The accurate use of ratios is based on the

Communicated by Klaas R. Westerterp/Håkan Westerblad.

\section{Coleman $(\triangle)$}

Canterbury Christ Church University, Canterbury, UK

e-mail: damian.coleman@canterbury.ac.uk

J. G. Hopker · L. Passfield

Endurance Research Group, School of Sport and Exercise

Sciences, University of Kent, Chatham, UK assumption that the slope of the relationship of the logarithmically transformed numerator and denominator is 1 ; if this is not the case then the ratio will scale inaccurately and lead to errors in the interpretation of measures at different absolute work rates. We are aware that the gross efficiency ratio does not scale appropriately across different work rates, and in line with recommendations, we rescaled values with a log-linked allometric model using the log of the energy expenditure as a covariate in the model. Between group differences for work rates were then assessed using a generalised linear model with energy expenditure (and also cadence) as covariates. We articulated these methods in our paper (Hopker et al. 2013) and in reply to Boning and Pries who had not fully appreciated this method of data analysis, which highlights the need to make colleagues aware of how to scale such comparisons.

The findings of the Brisswalter paper broadly support the findings of our paper that ageing reduces gross efficiency (Hopker et al. 2013). However, we believe the magnitude of the differences, and where those differences occur will be altered by the employment of these methods, which is fundamental to the utilisation of these data in the design of future studies in this area.

\section{References}

Boning D, Pries AR (2013) Pitfalls of efficiency determination in cycling ergometry. J Appl Physiol 115:1862

Brisswalter J, Wu SSX, Sultana F, Bernard T, Abiss CR (2014) Age differences in efficiency of locomotion and maximal power output in well-trained triathletes. Eur J Appl Physiol. doi:10.1007/ s00421-014-2977-8

Hopker JG, Coleman DA, Gregson HC, Jobson SA, Von der Haar T, Wiles J, Passfield L (2013) The influence of training status, age, and muscle fiber type on cycling efficiency and endurance performance. J Appl Physiol 115:723-729 